\title{
A complete ray-trace analysis of the Mirage toy
}

\author{
Sriya Adhya, John Noé
}

Sriya Adhya, John W. Noé, "A complete ray-trace analysis of the Mirage toy," Proc. SPIE 9665, Tenth International Topical Meeting on Education and Training in Optics and Photonics, 966518 (3 June 2007); doi: $10.1117 / 12.2207520$

SPIE Event: Tenth International Topical Meeting on Education and Training in SPIE. Optics and Photonics, 2007, Ottawa, Ontario, Canada 


\title{
A Complete Ray-trace Analysis of the 'Mirage' Toy
}

\author{
Sriya Adhya and John Noé
}

\author{
Department of Physics and Astronomy, Stony Brook University, Stony Brook, NY 11794-3800 USA \\ Phone: (631) 632-4303, Fax: (631)632-8176, Email: John.Noe@stonybrook.edu
}

\begin{abstract}
The 'Mirage' (Opti-Gone International) is a well-known optics demonstration (PIRA index number 6A20.35) that uses two opposed concave mirrors to project a real image of a small object into space. We studied image formation in the Mirage by standard 2x2 matrix methods and by exact ray tracing, with particular attention to additional real images that can be observed when the mirror separation is increased beyond one focal length. We find that the three readily observed secondary images correspond to 4,6 , or 8 reflections, respectively, contrary to previous reports.
\end{abstract}

Keywords: optics demonstrations, ray trace analysis

\section{Introduction}

The 'Mirage' [1] is a well known optics demonstration [2] that is very popular in our laboratory and at open house events. It consists of two horizontal concave mirrors that work together to project a real image of a small object placed on the lower mirror through an aperture in the upper one. Visitors are fascinated by the realistic image that "floats in space" and are challenged and involved when they are asked what will happen when it is viewed through a magnifying glass or mirror, or illuminated by a laser beam.

A little-known feature of the Mirage is the appearance of additional real images when the distance between the two mirrors is uniformly increased by carefully raising the upper mirror without tilting it. Three such secondary images (which are alternately inverted or not inverted compared to the primary image) are clearly visible at additional mirror distances of $3.1,4.5$, and $5.3 \mathrm{~cm}$ [3]. While the first secondary image is nearly as clear as the primary one, the subsequent secondary images are increasingly dim, distorted and hard to discern.

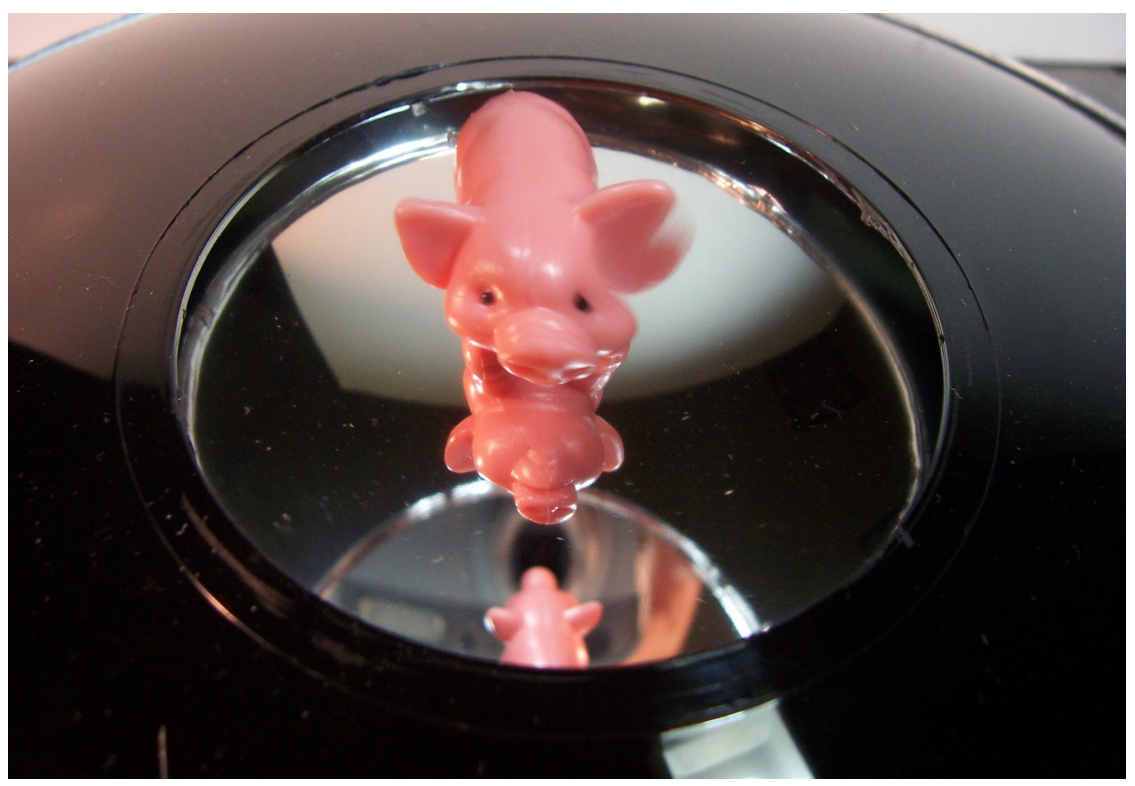

Fig.1. Photograph of the Mirage toy in our laboratory showing, from top to bottom, the projected real image, the projected reflection, and the actual object. The two images appear larger than the actual object primarily because the camera is close to the device. The opening in the upper mirror is $6.25 \mathrm{~cm}$ in diameter.

Tenth International Topical Meeting on Education and Training in Optics and Photonics, edited by Marc Nantel, Proc. of SPIE Vol. 9665, 966518 - C 2007 SPIE, OSA, IEEE, ICO doi: $10.1117 / 12.2207520$ 
We have studied the formation of these secondary images in the Mirage toy by both $2 \times 2$ matrix ray-optics methods in Mathematica [4] and by exact ray tracing with the BEAM2 program [5]. The problem provides a very good introduction to geometrical optics and to these two very useful and pedagogically valuable software tools. The project is typical of those used to introduce high school and young university students to optics at the Stony Brook Laser Teaching Center, as discussed elsewhere at this conference [6,7].

\section{Description}

The Mirage toy is readily available from various suppliers of science education products for about US $\$ 35$. Its two parts are made from a durable black plastic by injection molding. The mirror surfaces appear to be optically quite accurate except for an irregular portion $\sim 2 \mathrm{~mm}$ in diameter at the center of the lower mirror. This imperfection is of no consequence as it is normally covered by the object placed there. The optical surfaces are protected from tarnishing by a durable over-coating, but are quite sensitive to damage from fingerprints or scratches. We have found that cleaning is best done by wetting the surface with dilute household detergent and flushing with a copious stream of hot water; residual water droplets can be blotted dry with a paper towel, but the surface must never be wiped or rubbed. Now that the original Mirage patent has expired similar devices are being produced and sold by companies other than Opti-Gone. The ones we have seen are of significantly lower optical quality than the authentic Mirage and don't clearly show the secondary images.

The description of the Mirage on its packaging and elsewhere is misleading in several ways. The trade-marked name Mirage has of course no relation to the refraction phenomenon by that name, and the term " 3 -D reflection hologram" is not accurate either. The packaging further states that "optical surfaces are crafted to $5 / 1,000,000,000$ of an inch." This figure (which corresponds to only about one Angstrom) was intended to be indicative of the extreme thinness of the aluminum reflective coating [8].

\section{History}

The Mirage has an interesting history [8] that begins with a chance observation by a custodial worker, Caliste Landry, in the physics department at the University of California, Santa Barbara some four decades ago. One day Landry happened to be cleaning a stack of large World War II surplus searchlight mirrors that had been stored away in a closet. (Such mirrors have a central aperture for the arc lamp support.) Landry was startled and fascinated to see a realistic illusion of "dust that couldn't be cleaned." He reported his observation to a young faculty member in the department, Virgil Elings [9], who quickly recognized the optical principles involved and the novelty and potential utility of devices of this type for displaying jewelry, etc.

Elings and Landry filed for a patent for an "Optical Display Device" in 1970, and it was granted two years later $[10,11]$. The patent is interesting for what it does and does not say about the optics of the device. For example, Figures 3 and 4 in the patent show the second solution for two reflections and the non-inverted secondary image after four reflections, respectively. (See discussion in Section 5 below.) The accuracy of the images is not discussed, and the drawings show only one symmetrical pair of rays. The patent also mentions without any further discussion the possibility of using mirrors of unequal curvature or a mirror which isn't convex.

Michael Levin first became aware of the Elings device when he came across an expensive glass version of it (made by Elings and a son) at a San Francisco gift shop. Levin had prior experience with commercial ventures related to optics, having been involved with the Laserium shows [12] in the 1970's, and could see the potential of marketing a more affordable version to a wider audience. By 1977 he had acquired the rights to the Elings invention and founded Opti-Gone International, whose sole products remain the standard Mirage studied here (Model 2000) and a much larger version (Model 22) used for dramatic displays at museums and the like [1].

\section{Geometry}

We decided to study the geometry of the Mirage with no prior assumptions other than that the mirrors are surfaces of revolution. We first used a caliper and steel ruler to measure the active (coated) diameter of the lower mirror $\mathrm{c}$ and the perpendicular distance (sagitta) from the midpoint of that chord to the mirror surface $h$, taking care to account for the lip at the mirror's edge. From the relationship $r=c^{2} /(8 h)+h / 2$ we deduced a radius of curvature $r$ $=18.0 \mathrm{~cm}$. A circular template of this radius cut out from paper did not match the mirror surface, so it was apparent 
that this was not spherical. By trial and error it was determined that a smaller circular template $(\mathrm{r}=16.13 \mathrm{~cm})$ matched the surface well near its center, but deviated from it at the edges. Finally, a parabolic template with the same curvature at the vertex was constructed. It matched the entire surface well. Additional measurements confirmed that the vertices of the two mirrors in the Mirage are separated by one focal length $(8.06 \mathrm{~cm})$, as expected. The sagitta method was used to account for the large $(6.25 \mathrm{~cm}$ diameter) opening in the upper mirror.

It is of course clear why parabolic mirrors would be desirable, if not essential, in this device. A parabolic upper mirror placed one focal length above the lower mirror will direct all rays originating from the vertex of the lower mirror directly downwards, regardless of their angle with respect to the axis. When these precisely vertical rays reflect from parabolic lower mirror they will converge to an image point that lies at the vertex of the upper mirror, as shown in the following sketch taken from the Opti-Gone International web site [1].

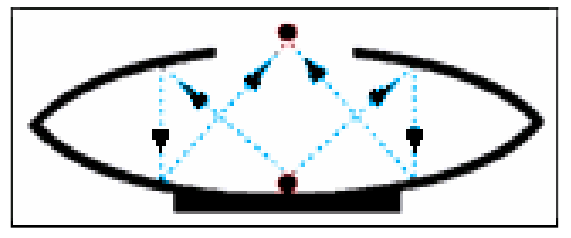

Fig. 2. Ray paths between parabolic mirrors [1].

\section{Matrix Analysis}

The $2 \times 2$ or $\mathrm{ABCD}$ matrix technique for ray tracing is well known $[13,14]$. The $(\mathrm{r}, \theta)$ form we used applies to spherical optical elements on a common axis, where the ray angle $\theta$ is sufficiently small that the paraxial approximation $\sin (\theta) \sim \tan (\theta) \sim \theta$ applies. This approximation is certainly not justified in the present situation, and in fact all rays close to the axis are lost through the opening of the upper mirror. The mirrors are also not spherical. Nevertheless it is an interesting and useful exercise to carry out the analysis and study the solutions obtained.

Our matrix analysis had two parts. First we studied the magnification and displacement of the primary image as a function of the displacement of the object above the lower mirror. Then we studied the secondary images by finding all mirror separations $\mathrm{d} / \mathrm{f}$ at which an image forms at the upper surface, after 2, 4, 6, 8, or 10 reflections.

The two optical elements represented by $2 \times 2$ matrices are the two mirrors of focal length $\mathrm{f}=8.06 \mathrm{~cm}$ and the varying drift distance $\mathrm{d}$ between them. A product matrix with elements $\mathrm{ABCD}$ is formed by multiplying the appropriate element matrices in sequence, from right to left. For the primary image formed by two reflections the sequence would be: drift (D), reflect (R), drift (D), reflect (R), drift (D). For four reflections the product matrix would include an additional RDR sequence, etc. The condition for the formation of a real image is that matrix element $\mathrm{B}=0$. When $\mathrm{B}=0$, the matrix element $\mathrm{A}$ gives the magnification.

The calculations were carried out with the popular Mathematica software tool [4], which was available to us at no cost through a campus license. (The free open-source program Scilab [15] would have been equally effective.) To locate the secondary images the product matrix for a particular case was written out and the values of drift distance $\mathrm{d}$ that make element $\mathrm{B}$ of this matrix zero were obtained. For $\mathrm{n}$ reflections there are $\mathrm{n}$ solutions, but many of these are for mirror separations less than the focal length, or occur at relatively large separations. The first solution for 2 reflections $(\mathrm{d}=\mathrm{f})$ is the primary image normally observed; a second solution occurs at $\mathrm{d}=3 \mathrm{f}$. This second solution has been discussed before by other authors [16,17], who erroneously associated it with the first secondary image.

Figures 2 and 3 and their accompanying captions summarize the results. The steadily increasing magnification and upwards shift of the primary image as the object is raised above the lower mirror surface is responsible for the slightly "muscular" appearance of the pig image. If one small pig is placed atop another, then the image of the upper one clearly appears larger than the lower. The plot of image locations shows interesting mathematical patterns. As the number of reflections increases to infinity a multitude of images will converge at the confocal distance $\mathrm{d} / \mathrm{f}=2.0$. 


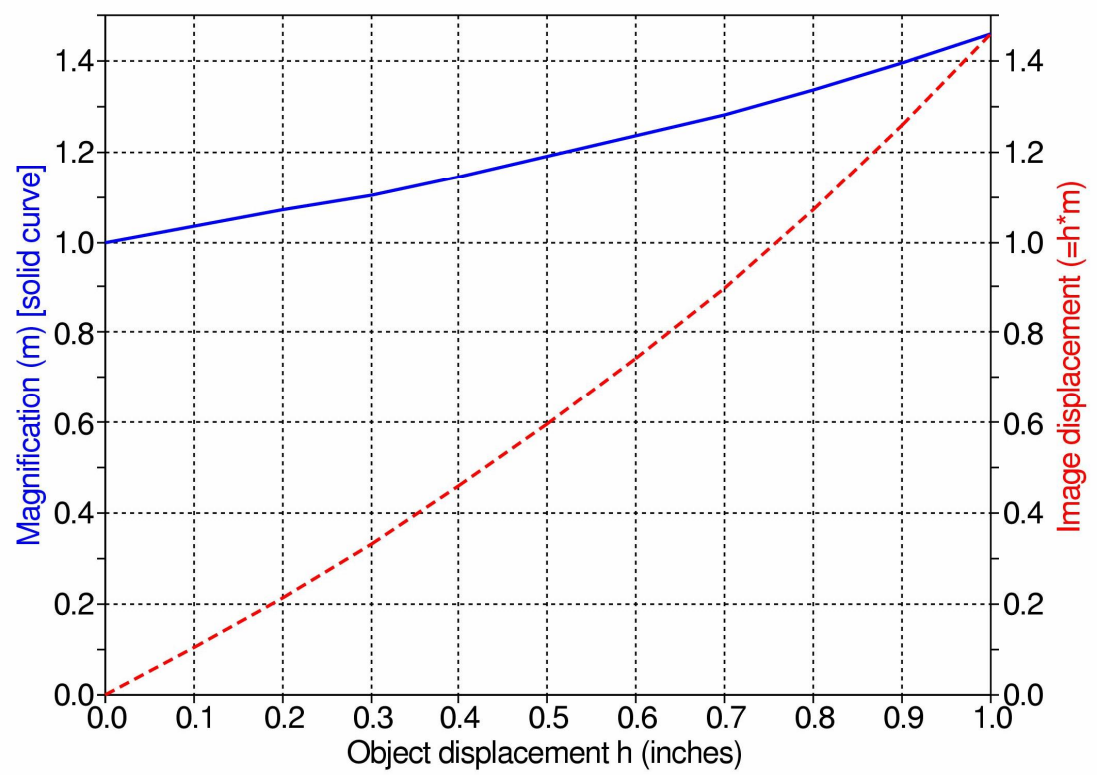

Fig. 3. Position and magnification of the primary image as a function of the object position relative to the lower mirror. As the object is displaced upwards its image expands and is displaced upwards by an amount that increasingly exceeds the shift in the object position.

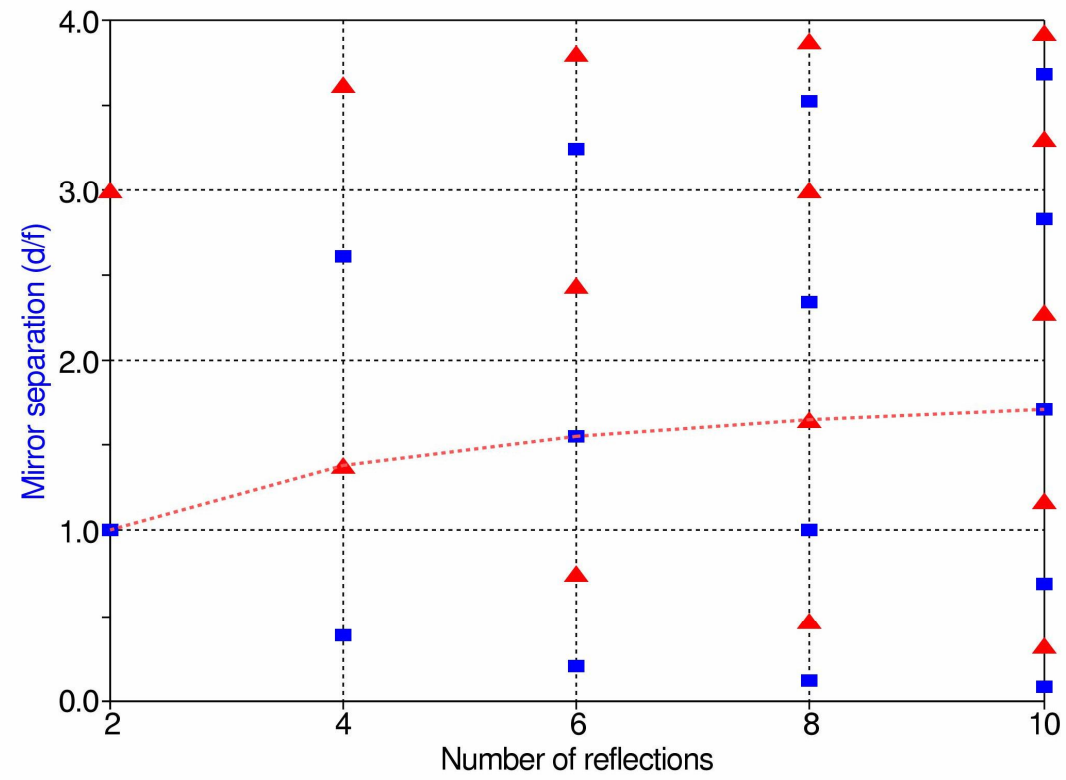

Fig. 4. This plot shows the locations of all images predicted by matrix analysis as a function of the mirror separation $d$ in units of the focal length $f$. The number of images is equal to the number of reflections, and the predicted images are either inverted (squares) or not (triangles). The dashed line shows the primary image and the observed sequence of secondary images. 


\section{Exact Ray Trace Analysis}

The BEAM2 software [5] proved to be an excellent tool for the exact ray trace studies, with limited yet quite adequate features for this study. (It only handles surfaces of revolution, but these can be formed by an arbitrary conic section.) It was quite easy to learn, especially with the detailed examples published by Atneosen and Feinberg [18]. Finally, it was quite affordable, just US $\$ 89$ for a single user copy, unchanged from 1991 [18].

We started by confirming that the primary image formed by parabolic mirrors one focal length apart is perfect, with no geometrical aberrations. The spherical aberrations produced by spherical mirrors are strikingly large, and even a few percent deviation from the optimum parabolic shape gives a noticeable imperfection in the calculated image. We next studied the first and clearest secondary image, produced by four reflections. Parabolic surfaces were assumed. As shown in Figure 5, a secondary image is formed, in agreement with the matrix analysis and observation, but it is clearly distorted by spherical aberration. Rays emerging from the object at relatively large angles cross the optical axis further from the object than do rays close to the axis. If only highly paraxial rays are considered the location of the image agrees precisely with the prediction of the matrix model (Figure 4), as it should. Not illustrated here, spherical aberration increases significantly with each additional pair of reflections,

We also looked at the two-reflection image predicted by the matrix model at a large mirror separation $\mathrm{d}=3 \mathrm{f}$. Once again, for rays nearly parallel to the axis (within a degree or less), an image is formed at the upper mirror, in agreement with the matrix model. However, as strikingly illustrated in Figure 6, realistic rays fail to converge, and may even remain parallel to the axis. Thus the exact BEAM2 calculation is in agreement with the easily done experiment - there simply is no observable two-reflection secondary image, as previously claimed $[16,17]$.

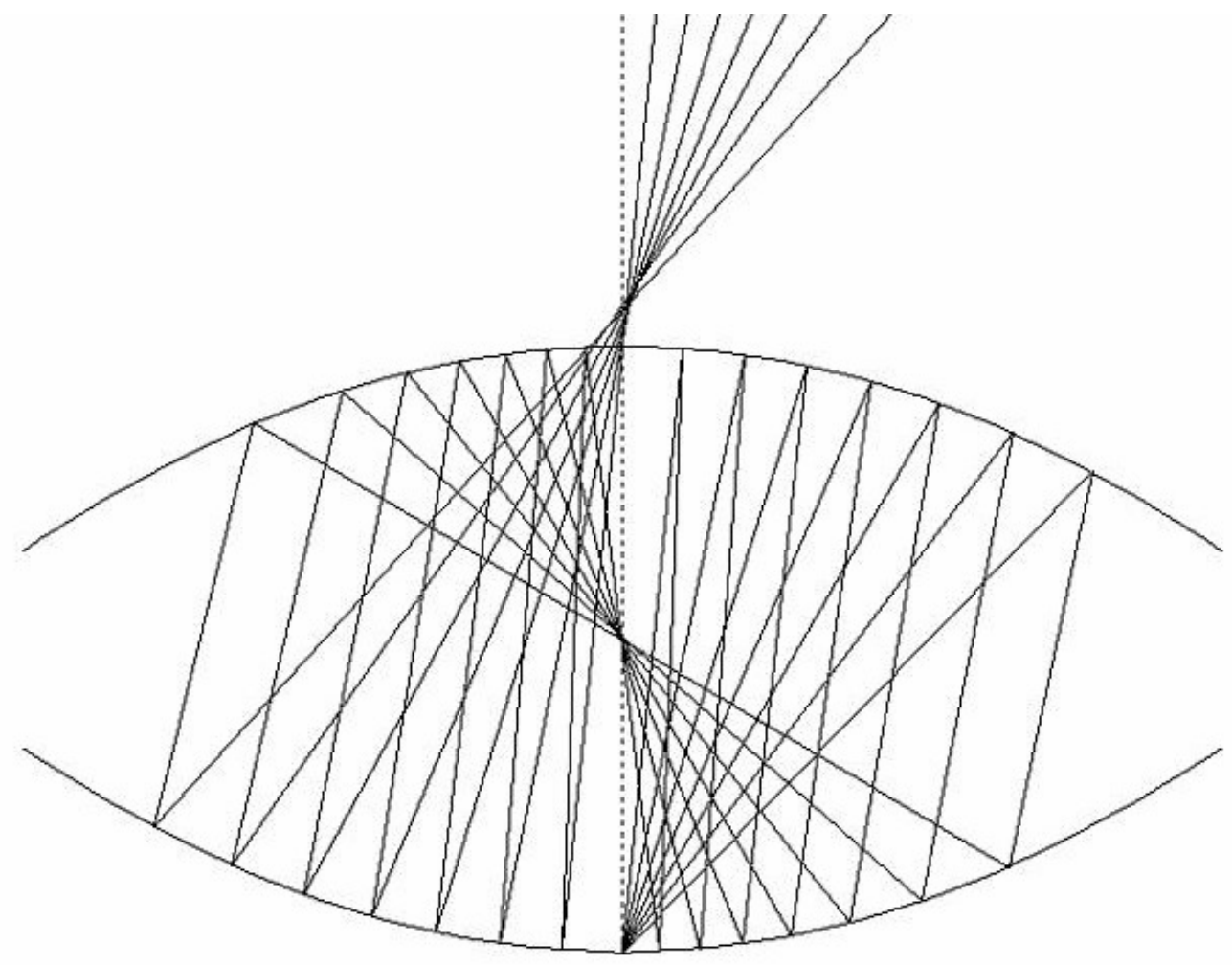

Fig.5. Exact ray trace for the first secondary image, calculated with BEAM2. An image is formed at the surface of the second mirror, but it is distorted by spherical aberration. The opening in the upper mirror is not shown, and some of the rays drawn would escape through it and not be reflected. 


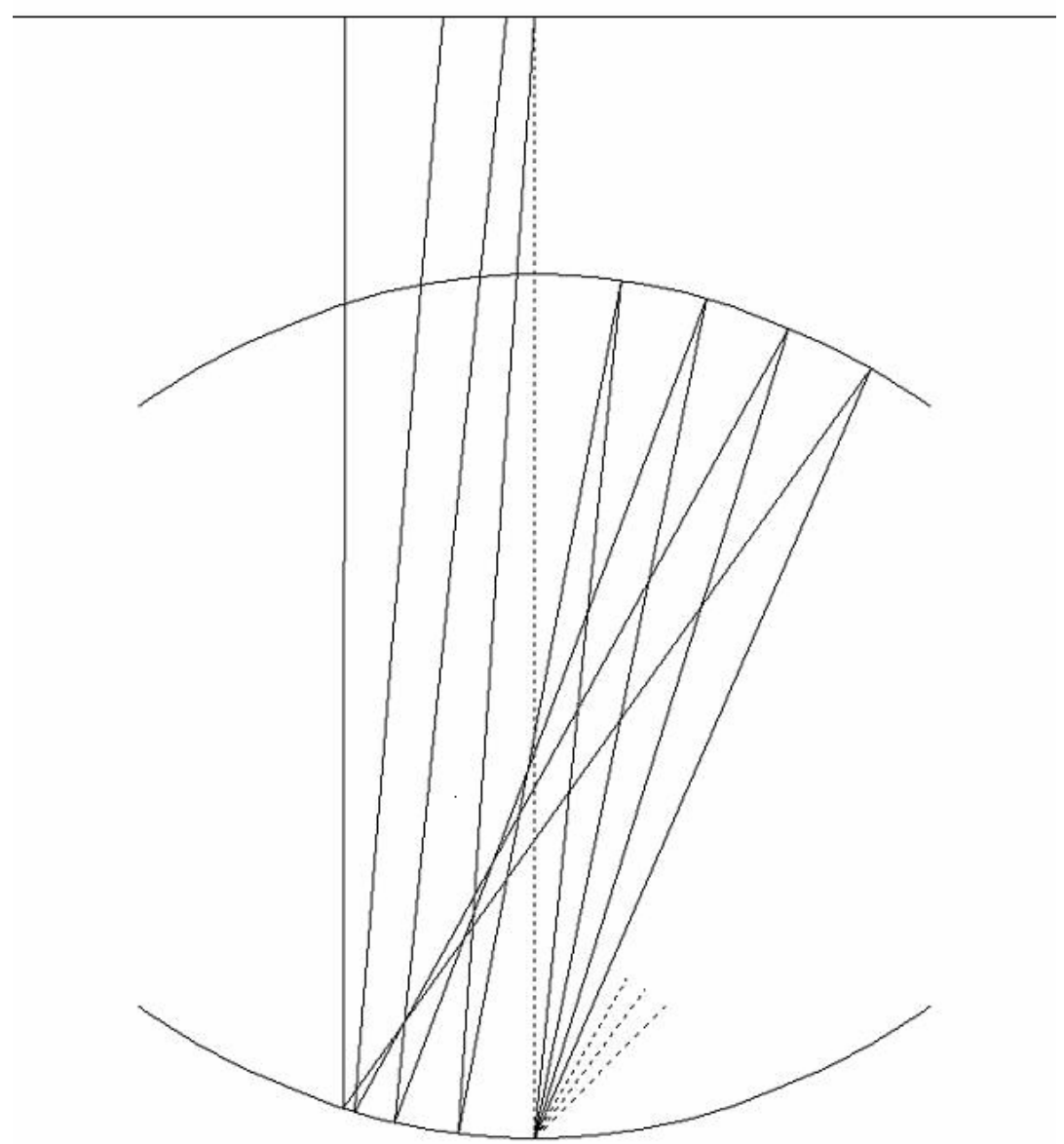

Fig. 6. Exact ray trace for a mirror separation $d=3 f$. The emerging rays do not come to a focus and there is no visible image. The horizontal line at the top is a "screen" that must be included when setting up the program.

\section{Discussion}

This turned out to be an excellent student project for a variety of reasons. The easily observed and interesting phenomena of the Mirage device provided a strong motivation for mastering new analytical tools. The mathematics and computer skills involved are relatively simple and well-suited to self study. Learning about the history of the Mirage and the people involved was an adventure, as was finding that published information was incorrect.

As with all good Laser Teaching Center projects [6,7], there is much more to learn and explore, through both simulations and hands-on measurements and experiments. As suggested in the patent filing there are many more optical configurations for image projectors that could easily be explored by the methods described here, including having mirrors of different shapes and unequal curvature (focal length). It would be interesting as well to apply the realistic analysis to the eight-reflection image that is predicted to coincide with the primary image at $\mathrm{d} / \mathrm{f}=1.0$, as shown in Figure 4. Hands-on experiments could involve placing a small light source at the object position to more precisely track the images, or even creating a reflecting telescope with one of the mirrors and studying its properties. (An even easier experiment is to use one of the mirrors to focus sunlight, with dramatic results!) In short, the Mirage toy provides a rich playground for exploring image formation and geometrical optics.

Finally, a recent paper in The Physics Teacher gives instructions for making a cylindrical device that produces a somewhat similar effect [19]. The device is simple, effective, and very inexpensive to construct, but it could not be analyzed in the same elegant and informative way that the Mirage toy was analyzed here. 


\section{Acknowledgments}

We would like to thank Michael Levin (Opti-Gone International) and Michael Lampton (Stellar Software) for interesting and informative discussions. We are also indebted to Azure Hansen for the excellent picture of our Mirage toy, and for her invaluable help with the manuscript. This work was supported in part by the Women in Science and Engineering (WISE) program and the Department of Physics and Astronomy at Stony Brook.

\section{References}

[1] "Mirage" is a registered trademark of its manufacturer, Opti-Gone International, Ojai, CA 93023 USA. http://www.optigone.com.

[2] The Mirage toy is listed on the PIRA (Physics Instructional Resource Association) database of physics demonstrations as number 6A20.35. See, for example, http://www.physics.brown.edu/physics/demopages/Demo/optics/demo/6a2035.htm.

[3] We obtained these results by supporting the upper mirror in a fixed horizontal position from its edges. The lower mirror was placed on a lab jack and slowly lowered to reveal the sequence of secondary images at a fixed viewpoint.

[4] Wolfram Research, Champaign, IL 61820-7237 USA, http://www.wolfram.com.

[5] BEAM2 ray-trace software, $\$ 89$ plus shipping. Stellar Software, Berkeley, California, http://www.stellarsoftware.com/.

[6] John Noé, "The Laser Teaching Center at Stony Brook University,” ETOP-2007, Ottawa (this conference).

[7] John Noé, "Simple Creative Projects from an Optics Teaching Laboratory,” ETOP-2007, Ottawa (this conference).

[8] Michael Levin, Opti-Gone International, private communication, May 2007.

[9] Virgil Elings went on to found Digital Instruments, Inc, the company which pioneered scanning probe microscopy (now part of Veeco, Inc.). There is an informative biography of Elings at http://web.mit.edu/physics/alumniandfriends/profiles/elings.html

[10] Virgil B. Elings and Caliste J. Landry, US Patent \#3647284, granted March 7, 1972. The full text of the patent is available from the Google Patents database at http://www.google.com/patents.

[11] This patent appears to have been Virgil Elings' first. It was followed by more than 40 others related to scanning probe microscopy.

[12] Laserium shows were developed by Ivan Dryer. The first was in November 1973 in Los Angeles. http://en.wikipedia.org/wiki/Ivan_Dryer

[13] A. Gerrard and J.M. Burch, Introduction to Matrix Methods in Optics. Dover Press, 1994.

[14] Eugene Hecht, Optics, Third Edition, Addison Wesley Longman, 1998.

[15] Scilab can be downloaded from http://www.scilab.org/.

[16] Andrzej Sieradzan, “Teaching Geometrical Optics with the 'Optic Mirage',” The Physics Teacher 28 534-536 (1990).

[17] Alex Dzierba, Indiana University. Course notes for Physics 360, http://dustbunny.physics.indiana.edu/ dzierba/P360n/notes/note4.pdf.

[18] Richard Atneosen and Richard Feinberg, "Learning optics with optical design software," Amer. J. Phys. 59 242-247 (1991).

[19] María Alicia Caussat, Héctor Rabal, Mikiya Muramatsu, "The Levitating Buddha: Constructing a Realistic Cylindrical Mirror Pseudo Image," The Physics Teacher 44 443-444 (2006). 